\title{
Dendritic Cells Feature Extraction using Geometric Features and 1D Fourier Descriptors
}

\author{
Anis Azwani Muhd Suberi ${ }^{\# 1}$, Wan Nurshazwani Wan Zakaria ${ }^{\# 2}$, Razali Tomari ${ }^{\# 3}$, Nurmiza Othman ${ }^{\# 4}$ \\ and Nik Farhan Nik Fuad ${ }^{* 5}$ \\ \#Advance Mechatronic Research Group (ADMIRE), Faculty of Electrical and Electronic Engineering, \\ Universiti Tun Hussein Onn Malaysia, 86400 Parit Raja, Batu Pahat, Malaysia \\ *UKM Medical Centre, Jalan Yaacob Latif, Bandar Tun Razak, 56000 Cheras, Kuala Lumpur, Malaysia \\ E-mail: ${ }^{1}$ anisazwani92@gmail.com, ${ }^{2}$ shazwani@uthm.edu.my, ${ }^{3}$ mdrazali@uthm.edu.my, ${ }^{4}$ nurmiza@uthm.edu.my, \\ 5nikfarhanfuad@gmail.com
}

\begin{abstract}
The current day technology such as Flow Cytometry is only able to classify Dendritic Cells (DCs) once they are stained. Subsequently this procedure affects the cell viability for vaccine preparation in DCs immunotherapy. Visually, the DCs classification can be distinguished through their unique morphological feature called tentacles compared to other immune cells, which have more rounded shape. Therefore, this paper proposes two pattern matching approaches based on Geometric and 1D Fourier Descriptors (FDs) to classify DCs from Phase Contrast Microscopy (PCM) image containing a mix of T-cells and debris. The performance of the developed algorithm is analysed and compared with the manual counting provided by the pathologist. The results show that the implementation of 1D FDs with Template Matching (TM) classifier have the better performance and achieve the best overall recognition accuracy of $\mathbf{9 8 . 3 \%}$ compared to Geometric features and DCCIS system.
\end{abstract}

Keywords - Dendritic cells; cancer immunotherapy; image processing; pattern recognition; phase contrast microscopy

\section{INTRODUCTION}

In recent years, Dendritic Cells (DCs) immunotherapy can be considered as a promising treatment to cure cancer [1],[2]. DCs immunotherapy employs and activates DCs in Peripheral Blood Mononuclear Cells (PBMCs) from patient body to fight the cancer cells as illustrated in Fig. 1. DCs are one of the PBMCs subtypes that have become a key role of antigen delivery. Other than that, DCs have gradually evolve as a vital therapeutic component against cancer. Before DCs are stimulated for vaccine preparation, they must first be classified from a PBMCs sample containing a mix of T-cells, DCs and debris particles.

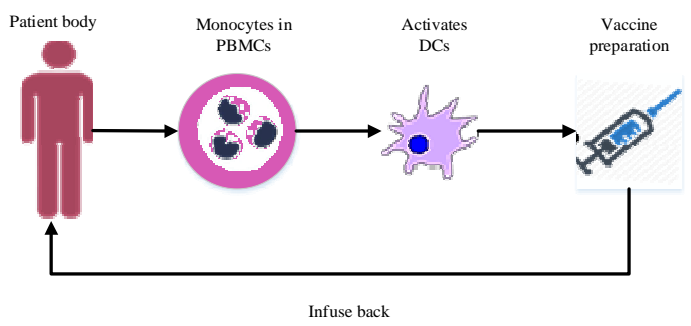

Fig. 1. DCs immunotherapy process
Till today, DCs classification is automatically quantified by using Flow Cytometry in which the DCs are fluorescently labelled [3],[4]. Although the utilization of this laser based tool is practical and rapid, DCs are unable to be labelled through any artificial means as fluorescence dyes used in the cell markers can contribute to the cell damage [4],[5]. Moreover, the result shows that the long processes of DCs culturing initiate a problematic for Flow Cytometry that produces an extensive amount of data and information, which can increase the complexity of the flow analysis [6]. Other possible approaches are investigated through image processing methods to identify DCs based on the morphology characteristic. DCs have a special characteristic with its long tentacle-like structures when compared to other immune cells as illustrated in Fig. 2 [7]. This feature characterizes them as a unique cell in which subsequently can be used to identify DCs automatically. This paper discusses two different DCs features that can be extracted which are 1) Geometric features and 2) One Dimensional (1D) Fourier Descriptors (FDs). 


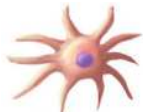

a) $\mathrm{DCs}$

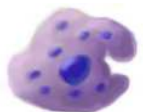

b) Macrophage

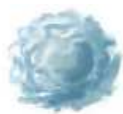

c) T-cell

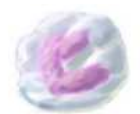

d) Monocyte

Most of current developed cell recognition system propose shape features such as circularity, compactness, area ratio and curvature to identify the target cell [8]-[11]. These features offer an effective way to detect rounded shape cell, however difficulties arise when it is use to detect a variation of unique cell shapes. Thus, a method on recognition of unique features such as dendritic spines has been implemented with morphological filtering and boundary extraction [12]. In addition, the dendritic spines have approximately similar unique shape with DCs. Therefore, cell shape signature can be one of the potential features in identifying DCs.

Generally, shape features are a set of numbers that represent the desired shape. In addition, the features are one of the well-known aspects in the human visual recognition system [13],[14]. The technique can be classified into two groups namely; contour based [15]-[17] and region-based methods [17], which compute shape features based on the boundary and extracts features from the whole region respectively. Shapes with associated features such as size, shape and orientation have drawn extensive attention in the scientific studies [10],[18],[19]. For example, basic Geometric features have been proposed in the classification of blood cells [20],[21]. Several features such as area, perimeter, diameter, circularity and others are computed and measured to classify the blood cell types into their classes. This method is simple and reduces the computational cost in the classification. However, a robust classifier is needed, as the cell shape is sensitive to different orientation. Thus, the target cell can be identified in whatever parameters control the cell shape.

The DCCIS system have been developed in which Artificial Neural Network (ANN) is used as a DCs classifier [22]. The accuracy achieved by the system is up to $90.1 \%$. However, the system involves redundant types of features such as area, solidity, eccentricity, circularity, medial axes ratio, deformation ratio, mean, standard deviation, skewness, kurtosis and entropy, which can increase the computational burden in the DCs classification. Instead of using complex classifiers, the recognition process also can be conducted using a simple approach called Template Matching (TM). This approach aims to search the most similar pattern between an image and a template image based on their information [23]. A normalized 2D cross-correlation pattern matching has been proposed in cell detection [24],[25]. The results show that the proposed method is able to recognise cells accurately in any of the imaging environment including normal, noisy and light-invariant environments.

Recently, Fourier Descriptors (FDs) are frequently being used in the cells recognition due to their invariance to translation, rotation and scaling [26]-[28]. Generally, shape descriptor can well match the corresponding cell if the target cell has a unique and superior shape discrimination. Also, it is the best methods for blood cell recognition as it achieves both representation and normalization very well [29]. All features used in the DCs identification system are described as follows. The details of the proposed framework are described in Section 2, Dendritic Cells (DCs) feature extraction, followed by the proposed system workflow in Section 3. Finally, the experimental result and conclusion is described in Section 4 and 5 respectively.

\section{MATERIAL AND METHOD}

\section{A. Dendritic Cells (DCs) Feature Extraction}

1) Geometric Features: Visually, the size and shape of DCs are bigger and irregular compared to other cells respectively [7]. Hence, the idea is to separate the other cells based on Geometric features in order to obtain the accurate DCs identification and counting results. Geometric features that are applied in this feature extraction process is shape factor and can be defined as Equation (1). This simple factor represents the cell shape in which the roundest cell has a higher value of area to perimeter ratio. The value is closer to 1 indicates that the cell is approximately round [30].

$$
\text { Shape factor }=\frac{4 \pi(\text { Area })}{(\text { Permeter })^{2}}
$$

Area and perimeter are calculated from each cell in the PBMCs image after the cell labelling stage. Area refers to the sum of the pixels that are enclosed inside the cell's boundary. Meanwhile, perimeter is the sum of boundary pixels that that outlining the cells. The geometric features have been calculated in pixel and are defined in Table 1. As expected, the circularity of T-cells shows a consistent value of 0.70 and above. Therefore, it can be proven that, T-cells have round and oval shapes since shape factor values are approximate to 1 .

TABLE I

Cell Classification ACCORding To CAlCulated Parameters

\begin{tabular}{|c|c|c|c|c|c|c|c|}
\hline Cell & $\boldsymbol{n}$ & \multicolumn{2}{|c|}{ Perimeter } & \multicolumn{2}{c|}{ Area } & \multicolumn{2}{c|}{ Shape factor } \\
\cline { 3 - 8 } & & Min & Max & Min & Max & Min & Max \\
\hline DCs & 40 & 215 & 770 & 2233 & 9096 & 0.19 & 0.61 \\
\hline T-cells & 40 & 55 & 198 & 169 & 2548 & 0.70 & 0.96 \\
\hline
\end{tabular}

2) $1 D$ Fourier Descriptors (FDs): The shape signature is widely used as a discriminative element in the cell recognition. Several studies have reported that shape descriptor based contour is one of the computation methods which has many advantages in recognizing geometric signatures, as it is easy to implement and promising a good matching performance [31],[32]. Therefore, 1D FDs is introduced as only DCs have the irregular shapes [33]. This is verified by the illustration of cell shape signatures in Fig. 3 [33]. The conversion of shape signatures starts with performing extraction of FDs on a contour, described by a vector of coordinates $\left(\boldsymbol{\theta}_{\mathrm{k}}\right)$. Next, Discrete Fourier Transform (DFT) is applied over the shape signatures to obtain the FDs coefficients as shown in Equation (2). 


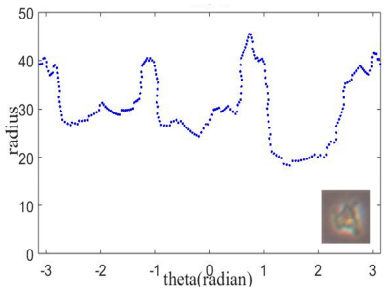

a)

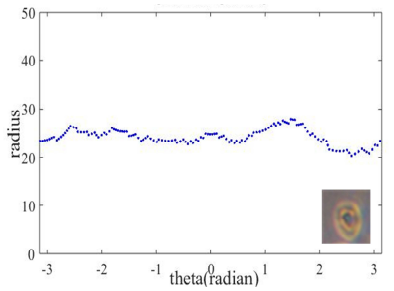

Fig. 3. Shape signatures of a) DCs and b) T-cells [33]

$$
\tilde{F}_{H}=F F T|U|=\sum_{k=0}^{N-1} U_{k} \exp \left(-\frac{2 \pi l}{N}\right) k \mu
$$

The phase information is neglected and only the magnitude values, $\tilde{F} \mu=|\tilde{F} \mu|$ is considered to achieve the orientation invariance of the Fourier coefficients. Since the FDs are symmetric, therefore only the first half are kept, $\tilde{F}(0) \ldots \tilde{F}(N / 2)$. Scale invariance is achieved by normalizing the FDs with respect to the magnitude of the DCs component, $\tilde{F} \mu=\tilde{F} \mu /|\tilde{F}(0)|$. By definition, the first normalized descriptor $F(0) / F(0)$ is equal to 1 and is neglected. Thus, the Fourier coefficients have been transformed into FDs based on translation, scaling and orientation invariances as demonstrated in Fig. 4 in order to maintain the system accuracy in different parameter that control the shape appearance [33]. In the case of an object contour, low frequency components of FDs contain information about the shape of the contour. In the meantime, the finer details along the contour are included in the higher frequency components [31].

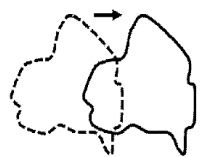

a)

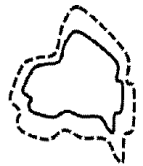

b)

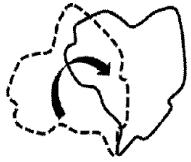

c)
Fig. 4. a) Translation, b) scaling and c) rotation invariants

\section{B. Proposed System}

Fig. 5 shows the proposed framework of DCs identification system. Two image dataset which consist of 145 images are retrieved from Cancer Research Malaysia (CRM) which have variations of light-variant environments and imaging artifacts. The PBMCs sample images are captured by Nikon, DS-Fi2 camera which attached to Olympus CK40 microscope (Phase Contrast Microscopy) with 100x and 200x magnifications. All the PCM images contain two populations of cells namely, DCs and T-cells (round shape) with particles called debris (black dots) as shown in Fig. 6.

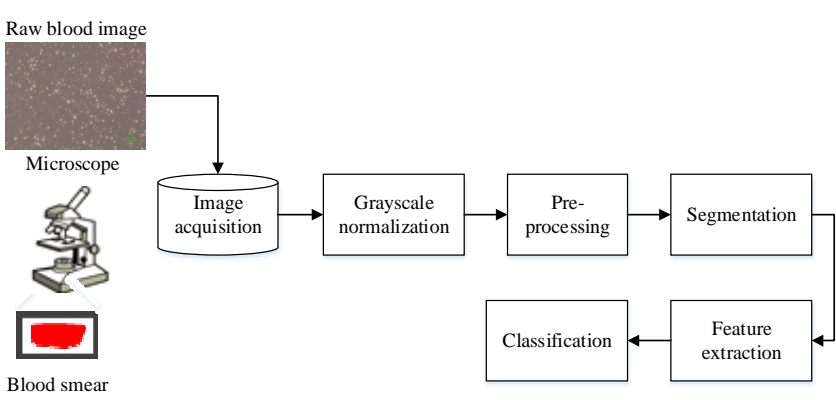

Fig. 5. Proposed framework of DCs identification

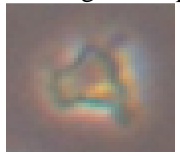

a)DCs

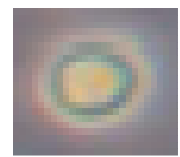

b)T-cell

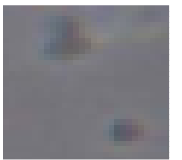

c)Debris
Fig. 6. Population of cells in PBMCs image

At the first stage, the image is converted into grayscale and normalized. Due to the imaging artifacts that deteriorate the PBMCs image, pre-processing stage is applied using $\mathrm{H}$ GLAT [34] and Halo Removal [35] methods to solve the overlapping cells and remove halo region that outlining the cells. Besides that, these two methods also diminish debris from the sample. Subsequently, several morphological operators such as clearing border, region fills, area opening and area closing are applied with Canny edge detector to acquire the cell contour in the segmentation stage [34]. The results are demonstrated in Fig. 7 [36].

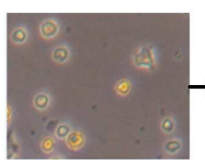

(a)

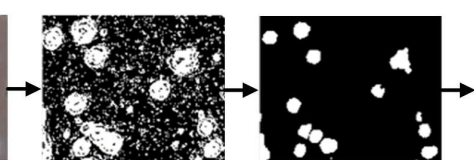

(b) (c)

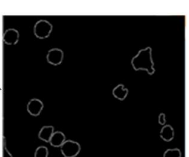

(d)
Fig. 7. a) Original image b) H-GLAT c) Halo Removal d) Segmentation [36]

After all, two classification frameworks using 1) Geometric features matching and 2) 1D FDs based on DCs templates are proposed as shown in Fig. 8 and 9 respectively. Geometric feature matching using the shape factor value of 0.70 has been applied to discriminate DCs from other cells. Any cell in the sample that has the value $<0.70$ indicates irregular shape and subsequently identified as DCs. Meanwhile, Template Matching (TM) method is applied to find the most approximate target cells based on the cell signatures of the DCs templates. Hence, the training session is conducted to select the most suitable DCs templates for DCs detection and minimize the recognition error between the DCs templates with target cells. In this classification flow, the templates are compared with testing images through sum of TM dissimilarity values in FDs. Here, smallest TM value in input image is considered as DCs. 


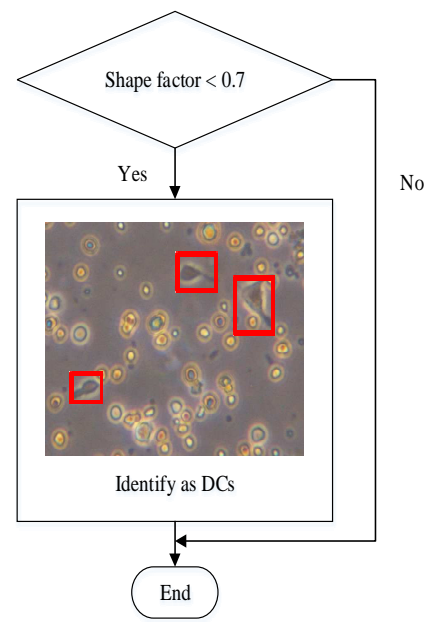

Fig. 8. Classification using Geometric features

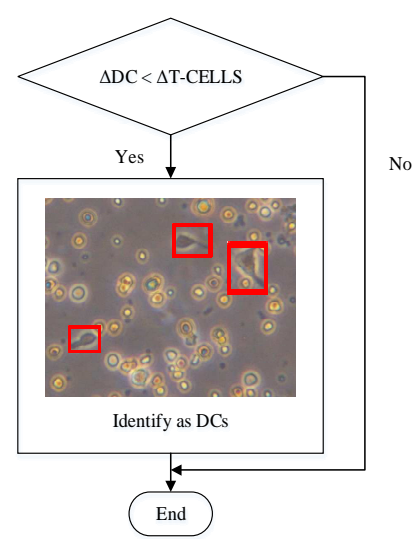

Fig. 9. Classification using 1D FDs and TM

\section{RESULTS AND DISCUSSION}

Both proposed methods are implemented, trained, and tested using MATLAB R2015a platform and runs on 2.5 $\mathrm{GHz}$ i3-3120M processor. The performance of both proposed methods are evaluated based on a) precision $(P), \mathrm{b})$ recall $(R)$ and c) accuracy $(A)$ as shown in Equation (3) below. $P$ refers to the exact amount of DCs and $R$ presents the exact number of DCs from the input image. Meanwhile, $A$ measures the overall system performance in classifying DCs.

$$
\begin{aligned}
& P=\frac{T P}{T P+F P}: R=\frac{T P}{T P+F N} \\
& A=\frac{T P+T N}{T P+F P+T N+F N}
\end{aligned}
$$

Table 2 shows some of the DCs classification results of Im_1 to Im_4 which are extracted from Dataset 1 with $1877 \times 1408$ pixels. The identified DCs of shape features and manual are marked with red marker and black arrow respectively. Meanwhile, the average processing time to run the system for each image in Geometric features and 1D FDs are 2.229 and 3.090 seconds respectively.

The performance results in terms of precision, recall and accuracy in Dataset 1 are tabulated in Table 3 and 4. Both shape features achieve accuracy and recall of $>90 \%$ for all images. However, lowest precision of Geometric features can be observed in Im_1, Im_2 and Im_3 with $33.3 \%, 66.7 \%$ and $40 \%$ respectively. Meanwhile, there have been an improvement for Im_1, Im_2 and Im_3 in terms of precision using 1D FDs. This is due to the implementation of invariance properties in which the descriptors are computed based on translation, scale and orientation invariances.

TABLE III

Comparison Between Geometric Features And 1D Fds In Dataset 1

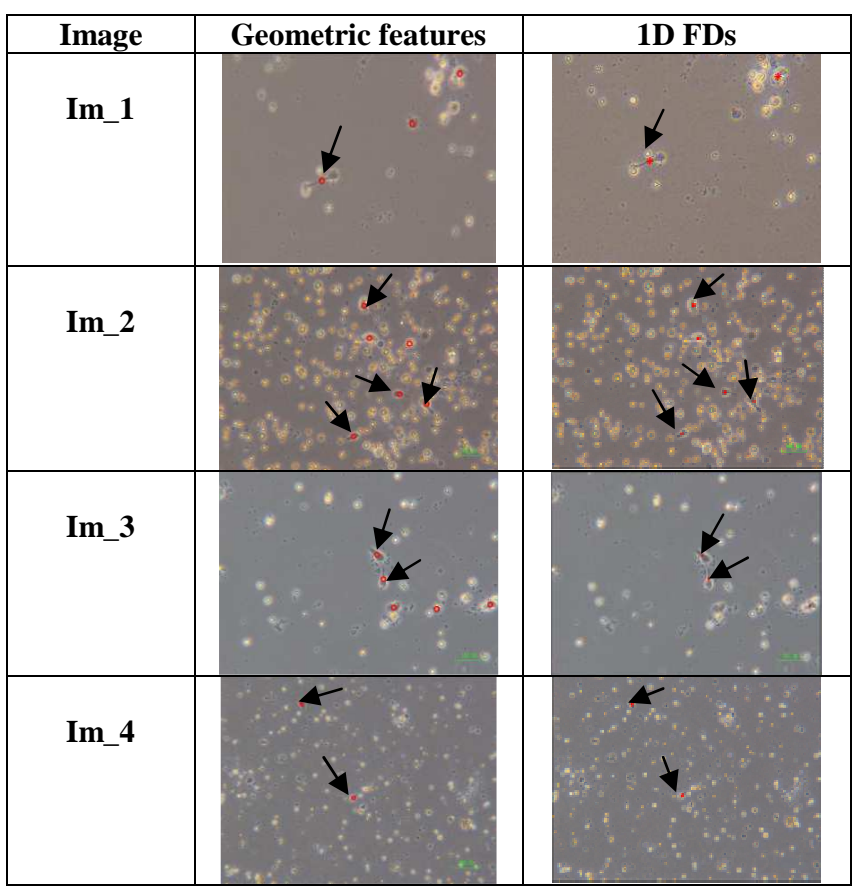

TABLE IIIII

Performance Of Geometric Features In Dataset 1

\begin{tabular}{|c|c|c|c|c|}
\hline Image & Im_1 & Im_2 & Im_3 & Im_4 \\
\hline TP & 1 & 4 & 2 & 2 \\
\hline TN & 27 & 194 & 30 & 120 \\
\hline FP & 2 & 2 & 3 & 0 \\
\hline FN & 0 & 0 & 0 & 0 \\
\hline Precision (\%) & 33.3 & 66.7 & 40 & 100 \\
\hline Recall (\%) & 100 & 100 & 100 & 100 \\
\hline Accuracy (\%) & 93.3 & 99 & 91.4 & 100 \\
\hline
\end{tabular}

TABLE IVV

PERFORMANCE OF 1D FDS In DATASET 1

\begin{tabular}{|c|c|c|c|c|}
\hline Image & Im_1 & Im_2 & Im_3 & Im_4 \\
\hline TP & 1 & 4 & 2 & 2 \\
\hline TN & 28 & 195 & 32 & 120 \\
\hline FP & 1 & 1 & 1 & 0 \\
\hline FN & 0 & 0 & 0 & 0 \\
\hline Precision (\%) & 50 & 80 & 66.7 & 100 \\
\hline Recall (\%) & 100 & 100 & 100 & 100 \\
\hline Accuracy (\%) & 96.7 & 99.5 & 97.1 & 100 \\
\hline
\end{tabular}

In contrast, the shape factor used in the Geometric features is sensitive to noise and distortions as there is no normalized features applied in the extracted features. Based on the precision results from both methods, the False Positive (FP) cases occur as there are some T-cells and debris, which appear in clumping conditions. Subsequently, 
these cells are visualised in the irregular shapes, which affect the DCs classification result. Therefore, specimen preparation procedure should be enhanced in order to diminish clumping particles such as debris that degrade the identification process.

The flexibility of Geometric features and 1D FDs to detect variance of cell shapes are also tested using second image dataset in Table 5 labelled Im_1 to Im_4 with pixel size of $2560 \times 1920$. There are various challenges in extracting DCs shapes from the PCM images. The first challenge is that the images consist of uneven background and the DCs are clumping to debris and T-cells. For the second challenge, some of the DCs tentacles are difficult to be observed. Generally, DCs have irregular shapes with varying length. It can be noted that some of the DCs tend to have an approximate shape with the clumping T-cells. "

TABLE V

COMPARISON BETWEEN GEOMETRIC FEATURES AND 1D FDS IN DATASET 2

\begin{tabular}{|c|c|c|}
\hline Image & Geometric features & 1D FDs \\
\hline Im_1 & & \\
\hline Im_2 & & \\
\hline Im_3 & & \\
\hline Im_4 & & \\
\hline
\end{tabular}

The performance for both shape features in Dataset 2 is tabulated in Table 6 and 7. Even though the accuracy is $>90 \%$ for all images, the system performance using Geometric features is quite poor. Based on the DCs identification results, the lowest precision of Geometric features can be found in Im_4 with $33.3 \%$. Other than that, both Im_2 and Im_4 shows the lowest recall percentage with the rate of $50 \%$. Meanwhile, for 1D FDs, the system performs $>70 \%$ of precision and recall in identifying DCs. Other than that, 1D FDs can provide up to $100 \%$ of precision, recall and accuracy specifically in Im_2. Hence, it can be proven that DCs are correctly identified with an acceptable error rate by using 1D FDs.

Toward the end, the performance evaluation of DCs classification also is conducted through comparison between the Geometric features, 1D FDs and DCCIS [22] as shown in Fig. 10. In DCCIS, the feature extraction incorporates 6 Geometric and 20 statistical features of colour moments using ANN classifier. Overall, Geometric features, 1D FDs and DCCIS achieve the best DCs classification accuracy of $95.9 \%, 98.3 \%$ and $90.1 \%$ respectively. The result of $1 \mathrm{D}$ FDs is slightly better than the Geometric features and DCCIS. Based on the results, it can be seen that all kind of shape features have vital contributions. Implementing 1D FDs is good enough as the DCs in the PBMCs image have distinctive shapes compared to other cells.

TABLE VI

Performance Of GeOMETRIC FeAtures In DATASET 2

\begin{tabular}{|c|c|c|c|c|}
\hline Image & Im_1 & Im_2 & Im_3 & Im_4 \\
\hline TP & 7 & 2 & 7 & 5 \\
\hline TN & 125 & 122 & 159 & 130 \\
\hline FP & 0 & 1 & 2 & 10 \\
\hline FN & 3 & 2 & 2 & 5 \\
\hline Precision (\%) & 100 & 66.7 & 77.8 & 33.3 \\
\hline Recall (\%) & 70 & 50 & 77.8 & 50 \\
\hline Accuracy (\%) & 97.8 & 97.6 & 97.6 & 90 \\
\hline
\end{tabular}

TABLE VII

PERFORMANCE OF 1D FDS IN DATASET 2

\begin{tabular}{|c|c|c|c|c|}
\hline Image & Im_1 & Im_2 & Im_3 & Im_4 \\
\hline TP & 8 & 4 & 9 & 10 \\
\hline TN & 123 & 123 & 158 & 135 \\
\hline FP & 1 & 0 & 3 & 4 \\
\hline FN & 3 & 0 & 0 & 1 \\
\hline Precision (\%) & 88.9 & 100 & 75 & 71.4 \\
\hline Recall (\%) & 72.7 & 100 & 100 & 90.9 \\
\hline Accuracy (\%) & 97 & 100 & 98.2 & 96.7 \\
\hline
\end{tabular}

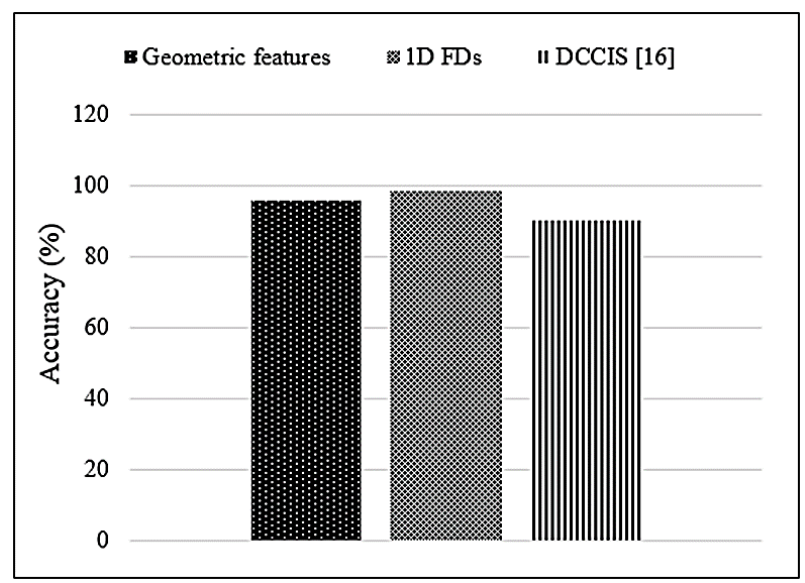

Fig. 10. Comparative performance of DCs classification

Although the accuracy of the 1D FDs is convincing, the performance measures in terms of precision still can be improved. The shape and statistical features provide a comparative performance. Hence, the correlation between these two features can be analysed and evaluated more details in the future. Other than that, the templates of DCs used can be updated to overcome the recognition error rate that can limit the performance.

\section{CONCLUSION}

An image-recognition system of DCs provides important benefits to assist clinical practitioners in enhancing the immunotherapy vaccine preparation. The development of a reliable and robust identification process for DCs is laborious as the PCM images are deteriorated with several imaging artifacts. The shapes of DCs are quite distinctive 
with other cells in which only DCs have irregular shapes. Therefore, shape features such as 1D Fourier Descriptors (FDs) and Geometric Features are introduced and evaluated to distinguish the DCs in the classification process. The result indicates that the discrimination power of 1D FDs with Template Matching (TM) is higher with $98.3 \%$ of accuracy compared to Geometric features and DCCIS system. This proposed method can be beneficial for identifying DCs as it accurately delineated the counted DCs for further analysis in automated live cell investigation. In future, the extracted features using 1D FDs can be integrated with texture features to resolve the misidentification of debris and other clumping cells as DCs.

\section{ACKNOWLEDGMENT}

This study is funded MOHE and UTHM by the Fundamental Research Grant Scheme (FRGS) Vot1583.

\section{REFERENCES}

[1] D. Raïch-Regué, M. Glancy, and A.W. Thomson, "Regulatory dendritic cell therapy: from rodents to clinical application," $J$ Immunology Letters, vol. 161, pp. 216-221, 2014.

[2] A. Sasada, M. Takagi, S. Tabata, M. Abe, and H. Abe, "A patient with stage IV gastric cancer who acquired complete remission after undergoing multi-peptide dendritic cell immunotherapy in combination with standard therapies," Personalized Medicine Universe, vol. 4, pp. 70-72, 2015.

[3] B. Rovati, S. Mariucci, M. Manzoni, K. Bencardino, and M. Danova, "Flow cytometric detection of circulating dendritic cells in healthy subjects," European J. of Histochemistry: EJH, vol. 52, pp. 45, 2008.

[4] Y.F. Tan, C.F Leong, and S.K. Cheong, "Observation of dendritic cell morphology under light, phase-contrast or confocal laser scanning microscopy," The Malaysian Journal of Pathology, vol. 32, pp. 97-102, 2010.

[5] A. Mohammadi, J. Mehrzad, M. Mahmoudi, M. Schneider, and A. Haghparast, "Effect of culture and maturation on human monocytederived dendritic Cell surface markers, necrosis and antigen binding," Biotechnic \& Histochemistry, vol. 90, pp. 445-452, 2015.

[6] R.R Jahan-Tigh, C. Ryan, G. Obermoser, and K. Schwarzenberger, "Flow cytometry," Journal of Investigative Dermatology, vol. 132, 2012.

[7] E. Wieder, Dendritic cells: A basic review, International Society for Cellular Therapy, 2003.

[8] H. Lee, and Y.P.P Chen, "Image based computer aided diagnosis system for cancer detection," Expert Systems with Applications, vol. 42, pp. 5356-5365, 2015.

[9] Q. Wang, L. Chang, M. Zhou, Q. Li, H. Liu, and F. Guo, "A spectral and morphologic method for white blood cell classification," Optics \& Laser Technology, vol. 84, pp. 144-148, 2016.

[10] S. Nazlibilek, D. Karacor, T. Ercan, M.H Sazli, O. Kalender and Y. Ege, "Automatic segmentation, counting, size determination and classification of white blood cells," Measurement, vol. 55, pp. 58-65, 2014.

[11] R. Tomari, W.N.W. Zakaria, M.M.A Jamil, F.M Nor, and N.F..N Fuad, "Computer aided system for red blood cell classification in blood smear image," Procedia Computer Science, vol. 42, pp. 206213, 2014.

[12] R. Su, C. Sun, C. Zhang, and T.D. Pham, "A novel method for dendritic spines detection based on directional morphological filter and shortest path," Computerized Medical Imaging and Graphics, vol. 38, pp. 793-802, 2014.

[13] M. Panwar, (2012) "Hand gesture recognition based on shape parameters," in Computing, Communication and Applications (ICCCA), 2012, p. 1-6.

[14] S. Venkatesha, and M. Turk, "Human activity recognition using local shape descriptors," in Pattern Recognition (ICPR), 2010, p. 37043707.

[15] Y. Xu, Y. Quan, Z. Zhang, H. Ji, C. Fermuller, M. Nishigaki, and D. Dementhon, "Contour-based recognition," in Computer Vision and Pattern Recognition (CVPR), 2012, p. 3402-3409.
[16] X. Bai, X. Yang, and L.J. Latecki, "Detection and recognition of contour parts based on shape similarity," Pattern Recognition, vol. 41, pp. 2189-2199, 2008.

[17] D. Chaudhuri, "Global Contour and Region Based Shape Analysis and Similarity Measures," Defence Science Journal, vol. 63, pp. 74, 2013.

[18] S. Chen, M. Zhao, G. Wu, C. Yao and J. Zhang, "Recent advances in morphological cell image analysis," Computational and mathematical methods in medicine, vol. 41, pp. 48, 2012.

[19] R. Tomari, W.N.W. Zakaria and R. Ngadengon, "An empirical framework for automatic red blood cell morphology identification and counting," ARPN Journal of Engineering and Applied Sciences, vol. 19, pp. 8894-8901, 2006.

[20] P.S Hiremath, P. Bannigidad, and S. Geeta, "Automated identification and classification of white blood cells (leukocytes) in digital microscopic images," IJCA Special Issue on "Recent Trends in Image Processing and Pattern Recognition" RTIPPR, pp. 59-63, 2010.

[21] M. Taherisadr, M. Nasirzonouzi, B. Baradaran, and A. Mehdizade, "New approach to red blood cell classification using morphological image processing," Shiraz E-Medical Journal, vol. 14, pp. 44-53, 2013.

[22] L. Yen Ruen, L. En Sheng, O. Hai Seng, and N.A. Mat Isa, "Dendritic cell classification imaging system," in Innovate Malaysia Design Conference. IMDCon, 2016, p.145-160.

[23] I.A. Aljarrah, A.S. Ghorab, and I.M. Khater, "Object recognition system using template matching based on signature and principal component analysis," Int. Journal of Digital Information and Wireless Communications (IJDIWC), vol. 2, pp. 156-163, 2012.

[24] H.K. Ra, H. Kim, H.J Yoon, S.H. Son, T. Park, and S. Moon, "A robust cell Counting approach based on a normalized 2D crosscorrelation scheme for in-line holographic images," Lab on a Chip, vol. 13, pp. 3398-3409, 2013.

[25] C. Chen, W. Wang, J.A. Ozolek, and G.K. Rohde, "A flexible and robust approach for segmenting cell nuclei from 2D microscopy images using supervised learning and template matching," Cytometry Part A, vol. 83, pp. 495-507, 2013.

[26] S. Bhonsle, and A. Klinzmann, and F. Park, "Centroid Distance Function and the Fourier Descriptor with Applications to Cancer Cell Clustering," Univ. of California, Undergraduate Rep., 2011.

[27] M.S. Kang, J.E Lee, H.R Kim, and M.H. Kim M.H, "Classification of tumor cells in phase-contrast microscopy image using fourier descriptor," Journal of Biomedical Engineering Research, vol. 33, pp. 169-176, 2012.

[28] M. Majurski, C. Zheng, J. Chalfoun, A. Dima, and M. Brady, "Comparison of shape signature sub-sampling methods for cell tracking," in Bioimage Informatics Conference, 2015, p. 3-7.

[29] E. Avci, "A new blood cell recognition algorithm based on directed vector method," Journal of Applied Biological Sciences, vol. 9, pp. $18-20,2015$.

[30] H. Lee H, and Y.P.P Chen, "Cell morphology based classification for red cells in blood smear images," Pattern Recognition Letters, vol. 49, pp. 155-161, 2014.

[31] C.K. Feudjio, A. Tiedeu, M.L. Noubeg, M. Gordan, A. Vlaicu, and S. Domngang, "Extracting and smoothing contours in mammograms using fourier descriptors," Journal of Biomedical Science and Engineering, vol. 7, pp. 119-129, 2014.

[32] H. Y. Martono, "Local Color Voxel and Spatial Pattern for 3D Textured Recognition," in International Journal on Advanced Science, Engineering and Information Technology, 2017, p. 650-661.

[33] A.A.M. Suberi, W.N.W. Zakaria, R. Tomari, and M.X. Lau, "Dendritic cell recognition using template matching based on onedimensional (1d) fourier descriptors (FD)," in First International Workshop on Pattern Recognition, 2016, p. 64-69.

[34] A.A.M. Suberi, W.N.W. Zakaria, R. Tomari, and K.P. Lim, "Optimization of overlapping dendritic cell segmentation in phase contrast microscopy images," in IEEE EMBS Conference on Biomedical Engineering and Sciences, 2016, p. 246-250.

[35] N. Jaccard, L.D. Griffin, A. Keser, R.J. Macown, A. Super, F.S. Veraitch, and N. Szita, "Automated method for the rapid and precise estimation of adherent cell culture characteristics from phase contrast microscopy images," Biotechnology and Bioengineering, vol. 111, pp. 504-517, 2014.

[36] A.A.M Suberi, W.N.W. Zakaria, and R. Tomari, "Dendritic cell recognition in computer aided system for cancer immunotherapy," Procedia Computer Science, vol. 105C, pp. 177-182, 2016. 